\title{
Robotic Systems for Meteor Observing and Moon Impact Flashes Detection in Spain
}

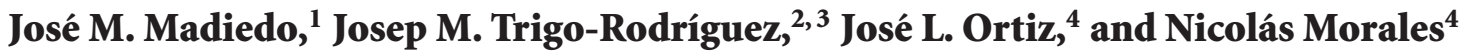 \\ ${ }^{1}$ Facultad de Ciencias Experimentales, Universidad de Huelva, Avenida de las Fuerzas Armadas S/N, 21071 Huelva, Spain \\ ${ }^{2}$ Institut d'Estudis Espacials de Catalunya, Gran Capità 2-4, Ed. Nexus, 08034 Barcelona, Spain \\ ${ }^{3}$ Institut de Ciències de l'Espai-CSIC, Campus UAB, Facultat de Ciències, Torre C5-p2., 08193 Bellaterra, Spain \\ ${ }^{4}$ Instituto de Astrofísica de Andalucía-CSIC, Camino Bajo de Huetor 50, 18080 Granada, Spain \\ Correspondence should be addressed to José M. Madiedo, madiedo@uhu.es
}

Received 28 June 2009; Accepted 21 September 2009

Academic Editor: Alberto J. Castro-Tirado

Copyright ( $\odot 2010$ José M. Madiedo et al. This is an open access article distributed under the Creative Commons Attribution License, which permits unrestricted use, distribution, and reproduction in any medium, provided the original work is properly cited.

A robotic observatory has been setup in the south-west of Spain with the aim to study meteoroids interacting with the Earth's atmosphere and meteoroids impacting on the Moon's surface. This is achieved by using an array of high-sensitivity CCD video cameras and three automated Schmidt-Cassegrain telescopes. We summarize here the main characteristics of this new facility.

\section{Introduction}

Since November 2008 a new robotic observatory is being setup by the University of Huelva in the environment of the Doñana National Park, in the south-west of Spain. Most of the systems in this astronomical observatory operate within the framework of the Spanish Meteor Network (SPMN), which is an interdisciplinary project dedicated to study meteoroids streams and the interaction of these particles of interplanetary matter with the Earth's atmosphere. Climate conditions in this area provide over 320 clear nights per year, which makes this location ideal for this research project. One of the systems we use for this purpose is based on a network of video stations located in different places in Spain. These employ an array of high-sensitivity CCD video cameras to monitor the night sky. The first two of these stations in Spain were setup in Andalusia by the University of Huelva in during 2006, and one more video station was setup by the same university in this region in 2007 [1]. Since then, these systems have been improved with the development of dedicated software for automation and data reduction. In fact, software development has been one of our more intensive and productive areas since 2006, and several packages with a total of over $500.000 \mathrm{C}++$ code lines have been written. The configuration and characteristics of these video devices have been also improved continuously. This has resulted in a significant advance that has been made in the latest video station setup by the above-mentioned astronomical observatory in 2009, as in this case the meteor detection system is fully autonomous, although it is flexible enough to allow for in situ or remote operation when necessary. This is the first robotic video CCD station for meteor observing that has been installed in Spain.

On the other hand, an automated system for lunar impact flashes detection has been setup in collaboration with IAA-CSIC. This is based on three telescopes that monitor the impact of meteoroids on the surface of the Moon. An important synergy is expected from the results recorded by both systems.

\section{Robotic Meteor Observing Station}

The University of Huelva is a pioneer in the development of fixed and mobiles video CCD stations for meteor observing in Spain. Thus, the first two of these stations operating in this country were setup by this institution in Andalusia in 2006 and one more video station was setup by the same university during 2007 [1-3]. During the last three years significant data have been obtained in collaboration with other SPMN video stations that have been setup later on [3-6]. 


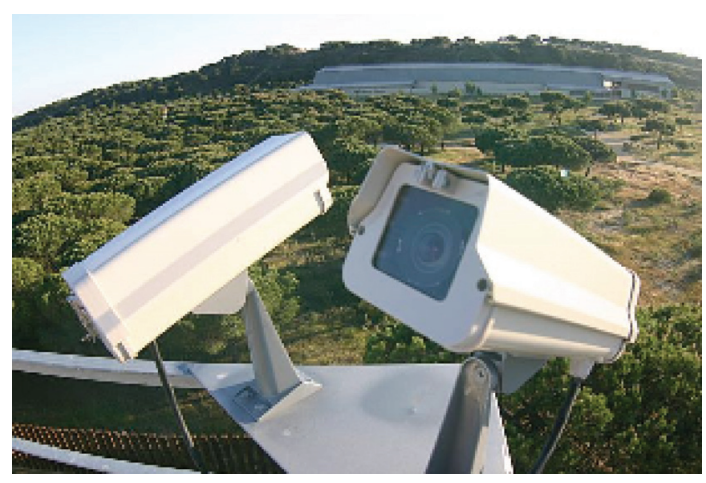

(a)

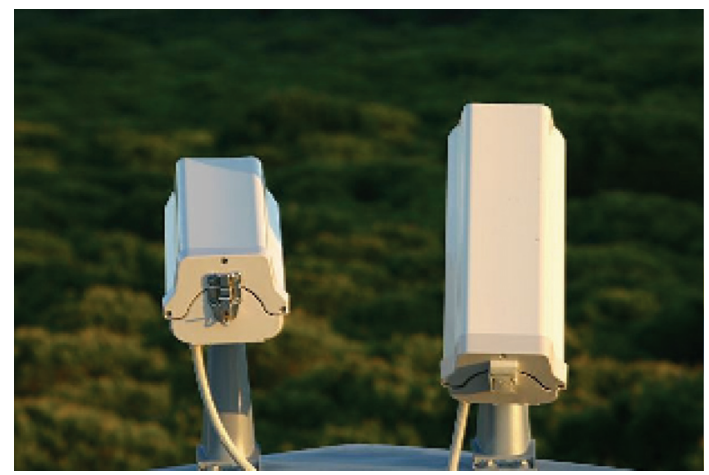

(c)

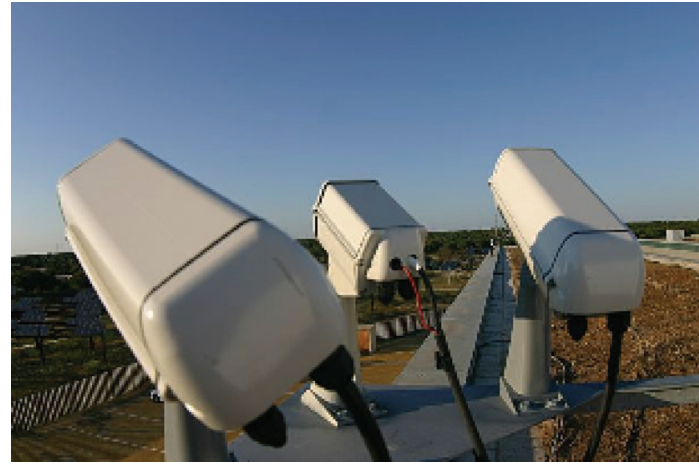

(b)

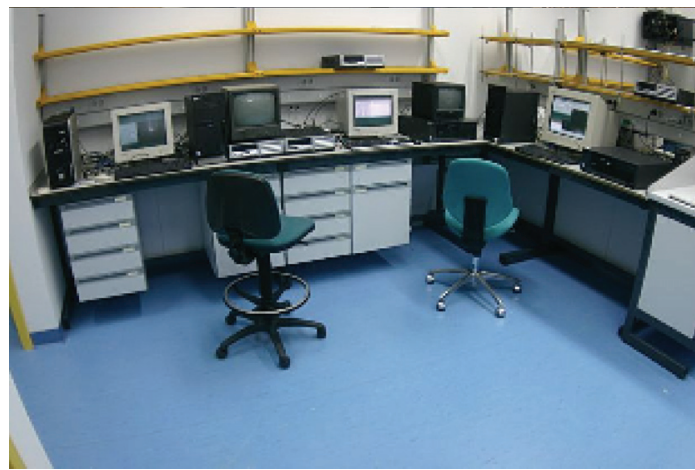

(d)

FIGURE 1: Images of the control room and some of the high-sensitivity CCD video cameras operating from the astronomical observatory setup by the University of Huelva.

Our new CCD video station is coordinated with the three above-mentioned ones and with several more SPMN stations that were setup later on in other locations in this country [1-3]. However, in this case the observing station has been configured to work in an autonomous way. It started operation in April 2009 and employs an array of 12 high-sensitivity Watec and Mintron CCD video cameras (Watec Corporation, Japan; Mintron Enterprises Co., Japan). Seven of them monitor the night sky and the other five operate during daytime (Figure 1). Nocturnal cameras are also endowed with holographic diffraction gratings (1000 grooves per millimeter) for obtaining meteor spectra. Fast aspherical lenses (f0.8) are attached to these cameras to maximize image quality and detect meteors as faint as magnitude $+2 /+3$ without using image intensifiers. Their focal length ranges from 3.8 to $6 \mathrm{~mm}$. Thermoelectric coolers are also attached to the cameras when operation temperature is above 25 degrees Celsius in order to improve the signal/noise ratio. The images taken by each camera at $25 \mathrm{fps}$ with a resolution of $720 \times 576$ pixels are continuously sent to a PC computer through a video capture card. The computers run a software package (UFOCapture, by SonotaCo, Japan) that automatically registers meteor trails and stores the corresponding video sequences on hard disk. This software includes time information on every video frame according to the current computer time. Each computer is automatically synchronized every 5 minutes with two redundant time servers that employ a GPS antenna. This allows us to measure time in a precise way (about 0.01 sec.) along the whole meteor path. The video cameras and the 12 personal computers that control the observing station are automatically switched on and off at predefined times, so that the system can operate without human intervention.

Once the observing session is over, one of the software packages developed in our, observatory scans every video file in order to identify very bright events (mag. -12 or brighter) and, if one of these is detected, an email is automatically sent to an operator. Another software also developed by the observatory automatically sends all the video files recorded during the observing session to an FTP server, from where they can be downloaded later on for data reduction. The astrometric measurements are then introduced into our recently developed Amalthea software that has been tested with Network software, which provides the equatorial coordinates of the meteors with a typical astrometric accuracy of about $0.01^{\circ}$ [7]. By the method of the intersection of planes, we reconstruct the trajectory and length of the meteor in the Earth's atmosphere for those events that have been simultaneously registered from at least two observing stations. Time information needed for the calculation of the initial velocity, average velocity, and deceleration is directly obtained from the video sequences. The orbital parameters 


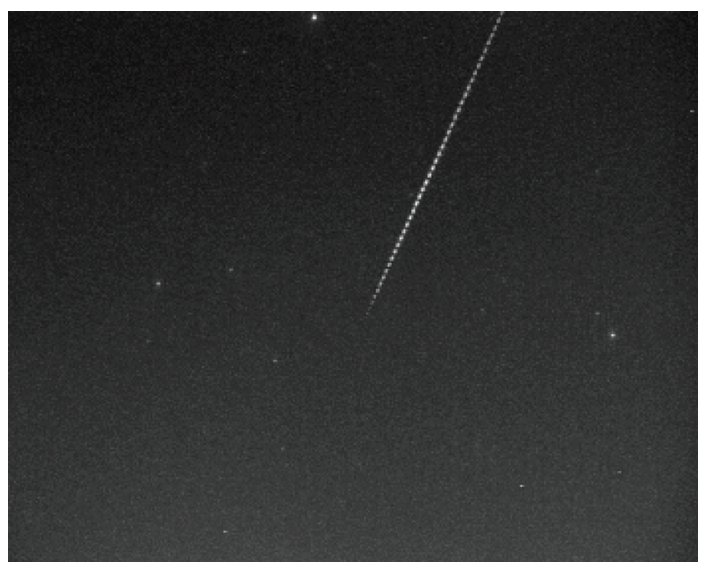

(a)

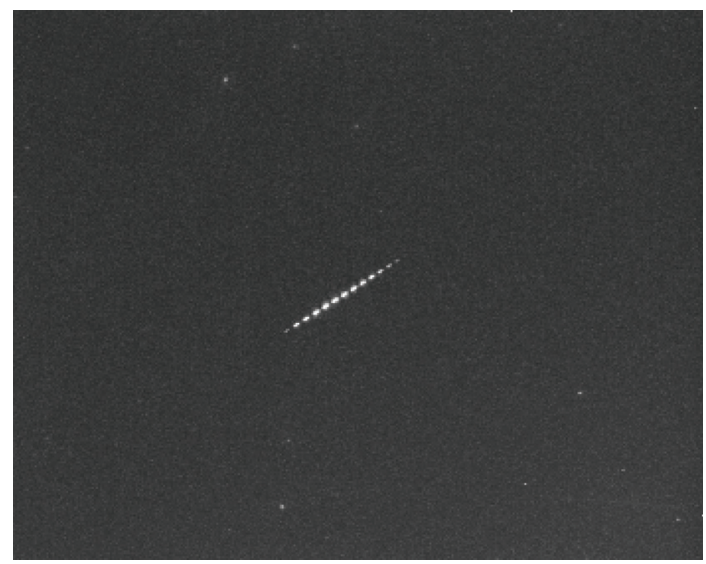

(c)

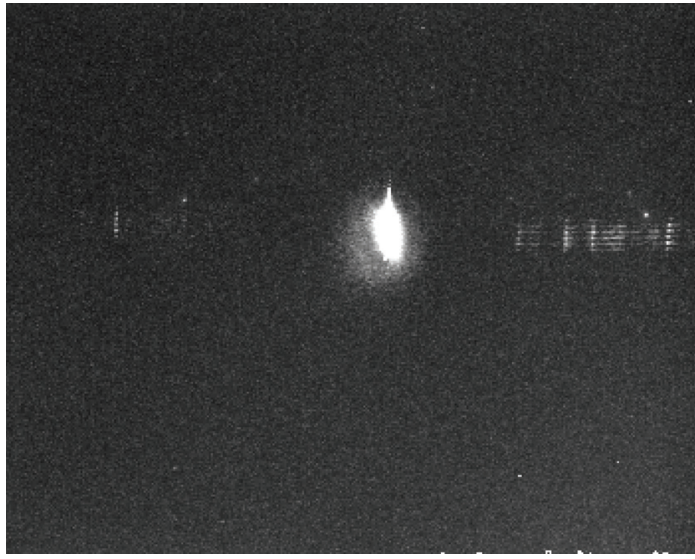

(b)

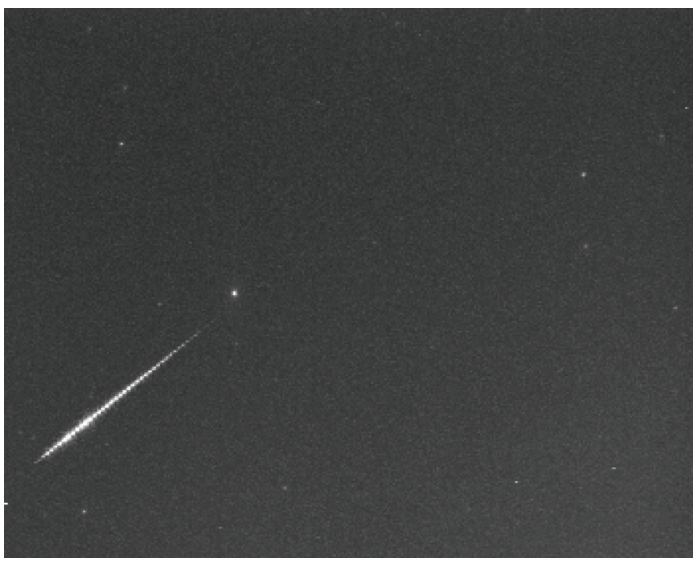

(d)

Figure 2: Low resolution mages of four of the meteors recorded by the robotic video CCD station. (a) Mag. 0 Virginid meteor registered on April 11, 2009 at 3 hours 37 minutes $34 \pm 0.01$ s UTC. (b) Mag. -8 Lyrid fireball registered on April 23, 2009 at 3 hours 24 minutes $07.49 \pm$ $0.01 \mathrm{~s}$ UTC. The emission spectrum can also be noticed on this image. (c) Mag. -1 sporadic meteor registered on April 27, 2009 at 4 hours 05 minutes $54.12 \pm 0.01$ s UTC. (d) Mag. -2.5 Alpha-Bootid meteor registered on April 28, 2009 at 0 hour 36 minutes $51.52 \pm 0.01$ s UTC.

of the corresponding meteoroid can be also obtained with Amalthea. For meteors brighter than mag. -6, the diffraction gratings attached to the video cameras operating during the night allow us to record the corresponding emission spectrum, which is also analyzed by the same software package in order to obtain information about the chemical composition of these particles. The parent bodies can be determined by means of our ORAS (ORbital Association Software) program, which employs several orbital similarity criteria to obtain this information [8-11].

Over 1200 meteor trails have been registered by this robotic video station during its first month of operation. Most of them are double-station events also registered from our video station in Sevilla, which is located at about $70 \mathrm{~km}$ from the new observatory. Figure 2 shows some of these events.

This new video station can be also remotely controlled by means of a standard web interface when necessary, as the different systems have been connected to a high-speed internet line (10 gigabit Ethernet). On the other hand, a high capacity UPS allows the observing station to keep on working during over six hours in the event of a power failure. If the UPS also fails or its batteries run out of energy, the operation of the video station is automatically resumed when power is available again. This is done thanks to a proper BIOS configuration of the computers connected to the video cameras and to a software package (called MetStationONOFF) that has been developed in this observatory to accomplish this task.

\section{Impact Flashes Detection System}

Two automated 14-inch SC telescopes have been setup in the astronomical observatory to monitor the impact of meteoroids on the surface of the Moon (Figure 3). Highsensitivity Watec CCD video cameras working at $25 \mathrm{fps}$ and with a resolution of $720 \times 576$ pixels have been attached to them in order to record the faint flashes produced by these impacts. A third 11-inch SC telescope will be also used from Sevilla, at about $70 \mathrm{~km}$ from the other two. This array of telescopes is intended to monitor the same area of the dark region of the Moon when the illuminated fraction of 


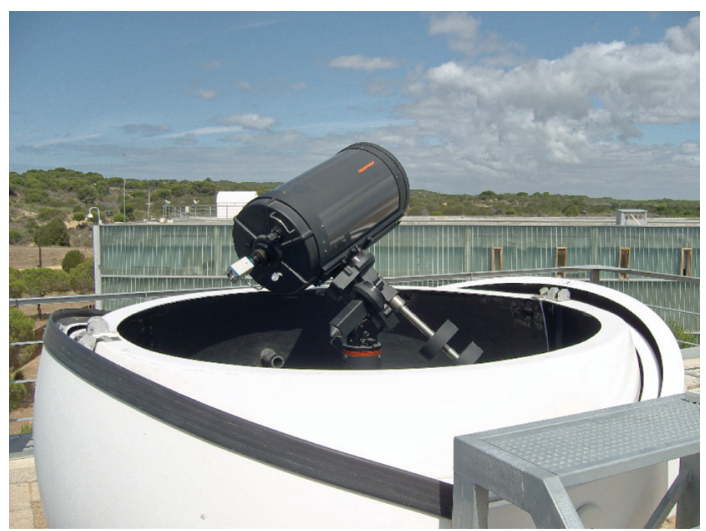

FIGURE 3: Image of one of the two 14-inch SC telescopes integrating the impact flashes detection system.

our natural satellite ranges from 0 to $60 \%$. By using several telescopes at the same time we can discard flashes produced by other sources, such as cosmic rays, space debris, satellites, and so forth [12]. The system has just been installed and is ready to start operation. It can be controlled in situ or remotely via a high-speed Internet connection.

The MIDAS software has been recently developed in our observatory to automatically identify lunar impact flashes produced by meteoroids. It allows for fast real time processing of the images obtained by the CCD video cameras attached to the telescopes. These images are sent from the cameras to the computers by means of a video capture card. The software also allows us to analyze video sequences previously recorded on hard disk or other media. GPS time inserters include time information on every video frame in order to measure time with a precision of about 0.01 second. These frames are continuously analyzed to identify the flashes, calculate the corresponding selenographyc coordinates of the impact, and estimate which is the likely origin (meteoroid shower and radiant) of the meteoroid. A photometric analysis is also performed in order to estimate the mass of the impactor.

Our software can also identify if a given flash has been produced by a meteoroid impacting on the Moon's surface and, so, if it must be analyzed or, on the contrary, if a flash has been produced by an artefact on the CCD of the corresponding video camera and, so, it has to be discarded. Thus, when a telescope detects an event it communicates with the other telescopes in the system via TCP/IP network protocol (up to 256 telescopes could be connected in this way by means of MIDAS). The other telescopes may then confirm or not if they have detected the same event. If the event is confirmed, it is automatically stored in a database for further processing. On the contrary, the event is ignored.

Although this system is fully operative and the impact flashes detection task works in an autonomous way thanks to the MIDAS software, further developments are necessary in order to achieve a fully robotic operation. Thus, for instance, some issues related to the improvement of lunar tracking must be solved.
This technique is very useful to provide valuable information about the flux of large meteoroids reaching the Earth [12]. It has the advantage that the area covered by the telescopes monitoring the surface of the Moon is much larger than the area covered by the systems that register the interaction of meteoroids with the Earth's atmosphere. Anyway, an important synergy is expected from the data recorded by both systems. Of special interest is, for instance, the identification of common events that would allow us to establish the possible existence of clusters of large meteoroids that might be impacting on the Moon and also producing very bright fireballs in the Earth's atmosphere that could give rise to meteorites.

\section{Conclusions}

A significant improvement has been made in our meteor network by setting up an autonomous CCD video station for meteor detection in the environment of the Doñana National Park, in the south-west of Spain. This has been mainly achieved thanks to several software packages that have been developed in our observatory since 2006. Software development has been also a very active task in the field of lunar impact flashes detection and a program called MIDAS, which is capable of real-time image processing, is available for this. An important synergy is expected from the results obtained by the lunar impacts detection system, which uses the Moon as a detector of meteoroids, and the meteor observing station, which uses the Earth's atmosphere for this purpose. Of special interest is, for instance, the detection of common events that might reveal the existence of associated meteoroids that could give rise to impacts on the Moon and also to very bright fireballs in our atmosphere or even to meteorite falls. These systems will also provide more accurate data related to the flux of interplanetary matter reaching the Earth. Optimal climate conditions in this area of Spain where the observatory is located (over 320 clear nights per year) will play an important role in this research.

\section{Acknowledgment}

The first author thanks IAA-CSIC for the support received to setup the Moon impact flashes detection devices.

\section{References}

[1] J. M. Madiedo and J. M. Trigo-Rodríguez, "Multi-station video orbits of minor meteor showers," Earth, Moon and Planets, vol. 102, no. 1-4, pp. 133-139, 2007.

[2] J. M. Trigo-Rodríguez, J. M. Madiedo, A. J. Castro-Tirado, et al., "Spanish meteor network: 2006 continuous monitoring results," WGN, Journal of the International Meteor Organization, vol. 35, no. 1, pp. 13-22, 2006.

[3] J. M. Trigo-Rodríguez, J. M. Madiedo, J. Llorca, et al., “The 2006 orionid outburst imaged by all-sky CCD cameras from Spain: meteoroid spatial fluxes and orbital elements," Monthly Notices of the Royal Astronomical Society, vol. 380, no. 1, pp. 126-132, 2007. 
[4] J. M. Trigo-Rodríguez, E. Lyytinen, D. C. Jones, et al., "Asteroid 2002 NY40 as a source of meteorite-dropping bolides," Monthly Notices of the Royal Astronomical Society, vol. 382, no. 4, pp. 1933-1939, 2007.

[5] J. M. Trigo-Rodríguez, J. M. Madiedo, I. P. Williams, et al., "The outburst of the $\kappa$-Cygnids in 2007: clues about the catastrophic break up of a comet to produce an Earth-crossing meteoroid stream," Monthly Notices of the Royal Astronomical Society, vol. 392, no. 1, pp. 367-375, 2008.

[6] J. M. Trigo-Rodríguez, J. M. Madiedo, I. P. Williams, et al., "Observations of a very bright fireball and its likely link with comet C/1919 Q2 Metcalf," Monthly Notices of the Royal Astronomical Society, vol. 394, no. 1, pp. 569-576, 2009.

[7] J. M. Trigo-Rodríguez and J. M. Madiedo Earth, "Determination of meteoroid orbits and spatial fluxes by using highresolution all-sky CCD cameras," Earth, Moon, and Planets, vol. 102, no. 1-4, pp. 231-240, 2008.

[8] R. B. Southworth and G. S. Hawkins, "Statistics of meteor streams," Smiths Control Astrophysics, vol. 7, pp. 261-285, 1963.

[9] J. D. Drummond, "A test of comet and meteor shower associations," Icarus, vol. 45, no. 3, pp. 545-553, 1981.

[10] G. Valsecchi, T. Jopek, and C. Froeschlé, "Meteoroid stream identification: a new approach. I. Theory," Monthly Notices of the Royal Astronomical Society, vol. 304, no. 4, pp. 743-750, 1999.

[11] P. Jenniskens, "Meteoroid streams that trace to candidate dormant comets," Icarus, vol. 194, no. 1, pp. 13-22, 2008.

[12] J. L. Ortiz, F. J. Aceituno, J. A. Quesada, et al., "Detection of sporadic impact flashes on the Moon: implications for the luminous efficiency of hypervelocity impacts and derived terrestrial impact rates," Icarus, vol. 184, no. 2, pp. 319-326, 2006. 

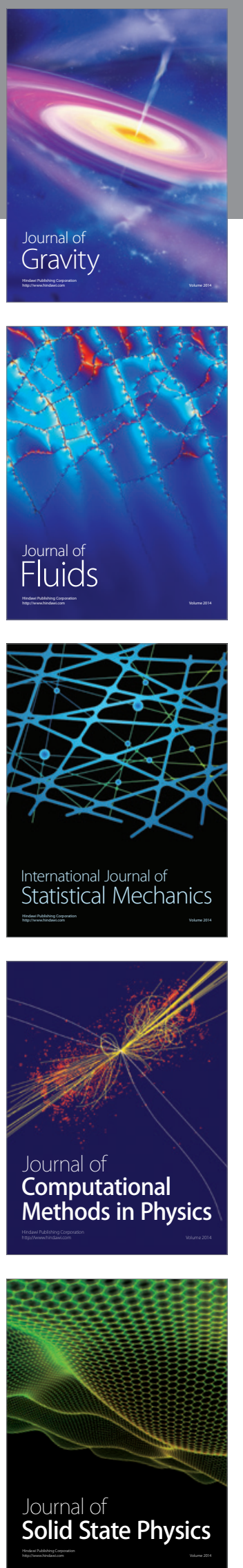

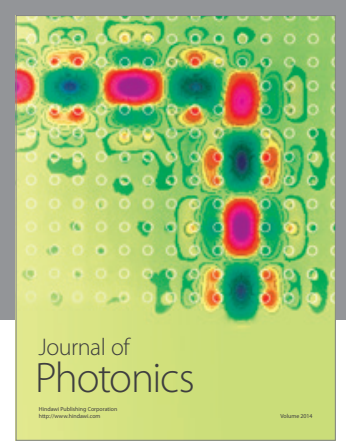

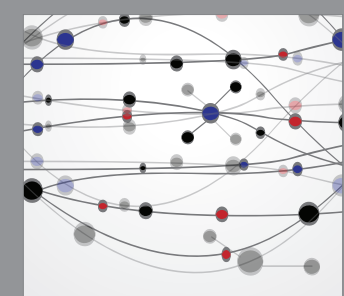

The Scientific World Journal
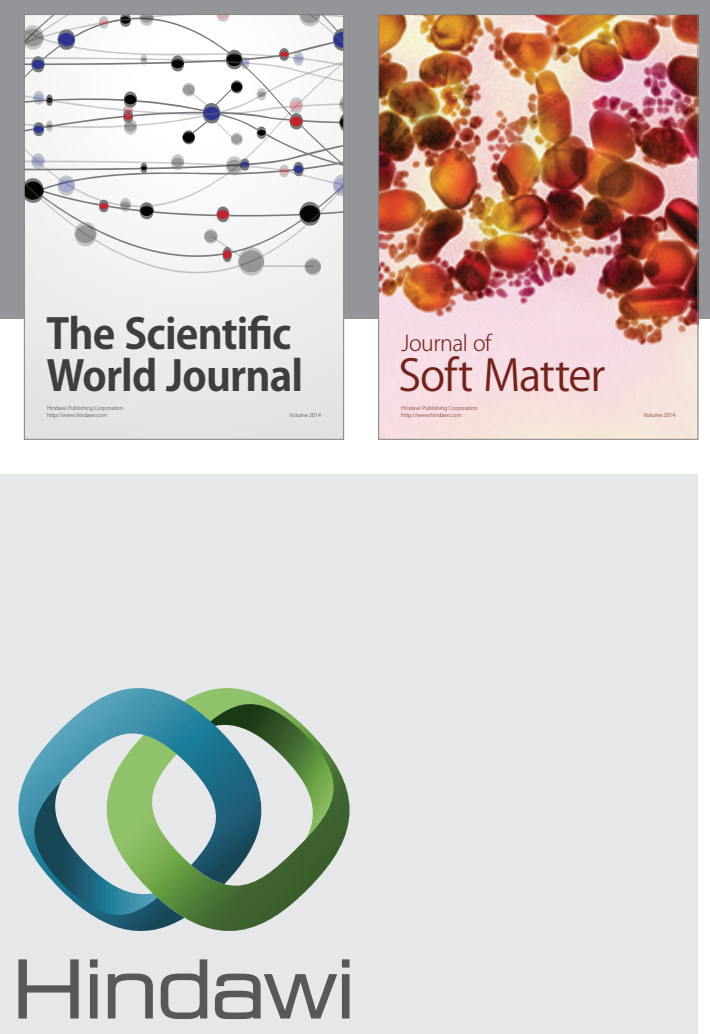

Submit your manuscripts at

http://www.hindawi.com
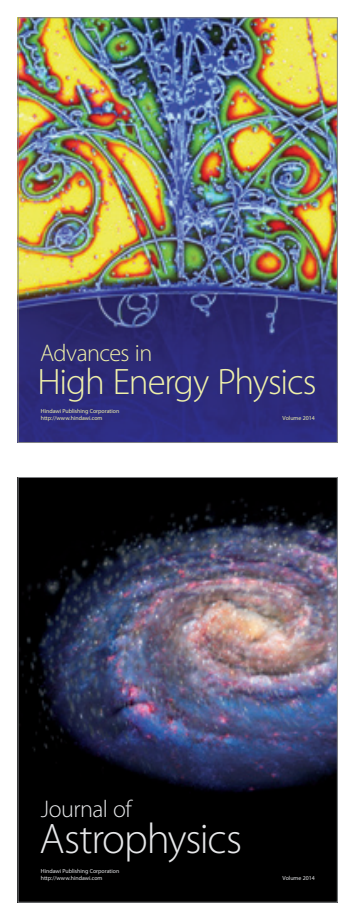
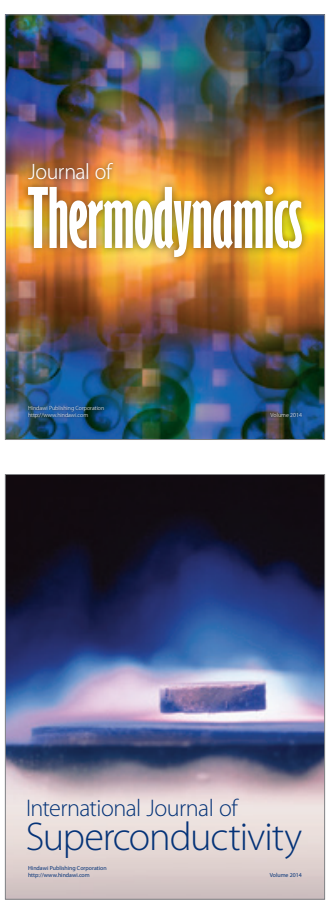
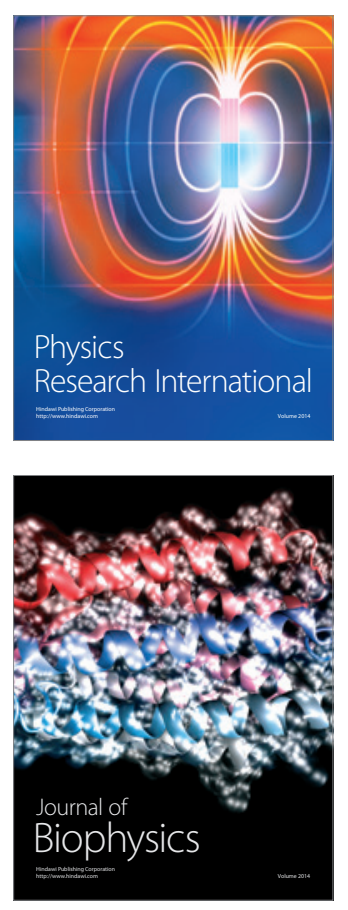
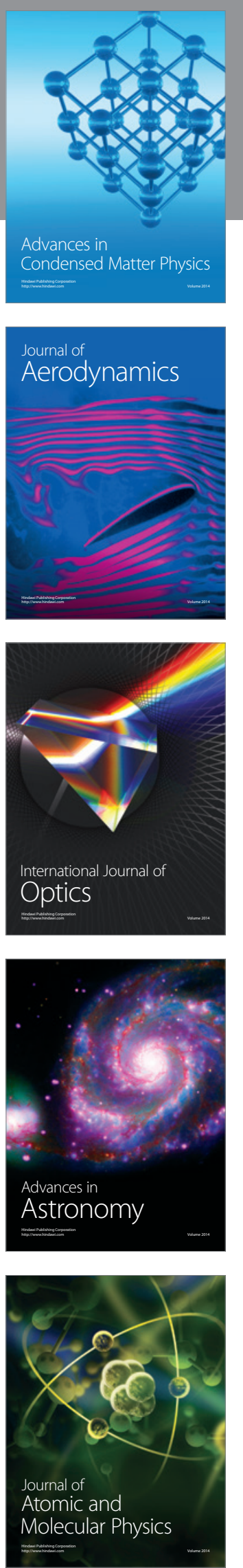